\title{
FILTROS ADAPTATIVOS IFIR: MODELAGEM VIA RESTRIÇÕES LINEARES
}

\author{
Orlando J. Tobias* \\ orlando@linse.ufsc.br
}

\author{
Rui Seara* \\ seara@linse.ufsc.br
}

*LINSE: Laboratório de Circuitos e Processamento de Sinais, Departamento de Engenharia Elétrica, Universidade Federal de Santa Catarina, Campus Universitário, 88040-900 - Florianópolis SC - Brasil

\begin{abstract}
This paper proposes an analytical model for describing the mean weight behavior of an adaptive interpolated FIR structure. The adaptive algorithm is the constrained filtered LMS, which is derived from the classical LMS algorithm. Since the interpolated FIR filter makes use of an interpolating structure cascaded with the adaptive filter, some assumptions used for the classical LMS can no longer be applied. The proposed model is obtained without considering the independence theory, which is a commonly used hypothesis to simplify the classical LMS analysis. In addition, by considering the adaptive interpolated filter topology a constrained analysis is introduced. Simulation results demonstrate the quality of the proposed model as compared to the ones available in the literature.
\end{abstract}

KEYWORDS: Adaptive filtering, adaptive interpolated FIR filters, LMS algorithm.

\section{RESUMO}

Este artigo propõe um modelo analítico para descrever o comportamento do valor médio dos coeficientes de uma estrutura adaptativa FIR interpolada. O algoritmo de adaptação utilizado é o LMS filtrado restrito, derivado do algoritmo LMS clássico. Como o filtro FIR interpolado emprega uma estrutura de interpolação em cascata com o filtro adaptativo, certas hipóteses assumidas para

\footnotetext{
Artigo submetido em 20/12/00

1a. Revisão em 09/04/02

Aceito sob recomendação do Ed. Assoc. Prof. Jacques Szczupak
}

o caso do LMS clássico já não podem mais ser aplicadas para essa particular topologia, devendo-se necessariamente usar outras considerações e aproximações. O modelo proposto é obtido sem considerar a teoria da independência, que é uma hipótese simplificativa bastante utilizada para a análise do LMS clássico. Baseandose na topologia do filtro adaptativo interpolado, é também desenvolvida uma análise utilizando restrições lineares. Resultados de simulações comprovam a qualidade do modelo proposto, quando comparados aos modelos disponíveis na literatura.

PALAVRAS-CHAVE: Filtragem adaptativa, filtros adaptativos FIR interpolados, algoritmo LMS.

\section{INTRODUÇÃO}

A partir do trabalho apresentado por Neuvo et alii (1984), no qual as estruturas FIR interpoladas (IFIR) foram introduzidas, uma grande quantidade de trabalhos têm sido desenvolvidos sobre esse assunto (Neuvo et alii, 1984; Neuvo et alii, 1987; Saramäki et alii, 1988). Atualmente, é fácil encontrar essas estruturas nas mais diversas áreas de aplicação, tais como: comunicações, instrumentação, dentre outras. A idéia básica desses filtros consiste em usufruir da redundância dos coeficientes do filtro FIR, permitindo remover algumas amostras de sua resposta ao impulso. As amostras extraídas podem ser então recriadas através de uma adequada estrutura de interpolação. Filtros FIR interpolados são especialmente atrativos visto que possuem todas as propriedades desejáveis dos filtros FIR, possibilitando uma mais eficiente implementação. Na literatura, são encontradas 
também algumas propostas de filtros IFIR adaptativos (Abousaada et alii, 1992; Seara et alii, 1992). Em algumas aplicações, as estruturas adaptativas FIR (AFIR) convencionais apresentam uma grande quantidade de coeficientes para adaptação, o que representa o principal inconveniente desse tipo de estruturas. Esse problema é originado nas operações necessárias para a atualização dos coeficientes e a filtragem propriamente dita. Nesse contexto é que os filtros IFIR adaptativos (AIFIR) podem representar uma alternativa eficiente para substituir as estruturas AFIR convencionais. Um filtro AIFIR possui todas as vantagens de uma estrutura IFIR, assim como as características desejáveis dos filtros AFIR convencionais. Por outro lado, é importante ressaltar que um melhor desempenho dessa estrutura fica sempre restrita às características do filtro interpolador utilizado. Isto é, a aplicação de estruturas adaptativas interpoladas é mais apropriada quando os sinais envolvidos apresentam seus componentes de freqüências limitados à banda passante do filtro interpolador. A maior parte dos trabalhos disponíveis envolvendo estruturas AIFIR se concentra na comparação do seu desempenho em relação às estruturas AFIR convencionais. Esses trabalhos têm sido importantes para determinar algumas propriedades dessas estruturas (Abousaada et alii, 1992; Seara et alii, 1992). Entretanto, é preciso contar com modelos analíticos que descrevam o comportamento do filtro, permitindo predizer seu desempenho para diferentes condições de funcionamento.

O objetivo deste trabalho é fornecer um embasamento teórico, sob o qual possam ser desenvolvidos e estudados modelos analíticos para o comportamento dos coeficientes de estruturas AIFIR que empregam o algoritmo LMS. O modelo é derivado utilizando considerações que não estão incluídas nas análises atualmente disponíveis. As análises desses tipos de estruturas até agora têm procurado estender para os filtros interpolados a teoria de análise do FIR-LMS clássico, não considerando as restrições a que alguns coeficientes do filtro estão submetidos. Isto é, a análise clássica considera que o espaço dos coeficientes é irrestrito, ou seja, os coeficientes de um dado filtro adaptativo podem assumir qualquer valor de forma a minimizar uma determinada função custo. Por outro lado, em uma estrutura AIFIR, apenas $N / L$ coeficientes dos $N$ possíveis são adaptados, sendo $L$ o fator de interpolação. Isso significa que o espaço de coeficientes de uma estrutura AIFIR é um subespaço da correspondente estrutura AFIR. Então, para realizar uma análise apropriada, devemos utilizar uma ferramenta matemática que permita incorporar essa restrição no processo de análise da estrutura AIFIR. Uma variante do algoritmo LMS, o algoritmo CLMS (constrained-LMS) (Frost, 1972) permite considerar essa situação e, assim, obter um modelo do comportamento dos coeficientes de um filtro AIFIR. Um outro ponto abordado está relacionado à teoria da independência (TI), que é uma hipótese simplificativa freqüentemente utilizada na análise de filtros adaptativos (Mazo, 1979; Widrow e Stearns, 1985; Haykin, 1996). Através da TI, considera-se que os vetores de entrada são estatisticamente independentes. Entretanto, a presença do filtro de interpolação faz com que os vetores em diferentes instantes sejam correlacionados. Com isso, a TI já não pode ser mais aplicada e, para uma correta análise, devemos considerar a correlação existente entre os vetores de entrada (Tobias et alii, 2000). Neste trabalho, foram consideradas tais correlações como também a inclusão de restrições no processo de análise, resultando em um modelo elegante e preciso para predizer a evolução do comportamento médio dos coeficientes da estrutura AIFIR-LMS estudada.

\section{FILTROS IFIR - FILTRO ÓTIMO}

A Figura 1 mostra o diagrama em blocos da estrutura clássica de um filtro IFIR com coeficientes fixos. Em Neuvo et alii (1984), Neuvo et alii (1987) e Saramäki et alii (1988), são apresentados diversos procedimentos para realizar o projeto de tais estruturas. No contexto de filtragem adaptativa, procura-se determinar o melhor conjunto de coeficientes para o filtro esparso adaptativo (W), de forma a minimizar uma determinada função custo.

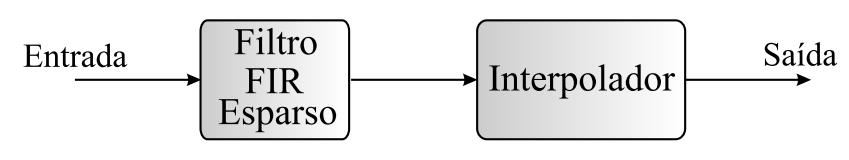

Figura 1: Diagrama em blocos de um filtro IFIR clássico.

Na Figura 2, W e I representam o filtro FIR esparso e o filtro interpolador, respectivamente. O sinal de entrada é denotado por $\mathbf{X}(n)=[x(n), x(n-1), \ldots, x(n-N+$ $1)]^{T}$. Para o esquema da Figura 2, assumimos que $x(n)$ e $d(n)$ são amostras de um processo aleatório, onde $d(n)$ é o sinal desejado. $y(n)$ e $e(n)$ representam a saída da estrutura interpolada e o erro de estimação, respectivamente.

A função tipicamente utilizada para determinar o vetor de coeficientes ótimo é o erro quadrático médio. Considerando-se a particular topologia da estrutura adaptativa abordada neste trabalho, representada pela esparsidade do vetor $\mathbf{W}$, deve-se buscar uma nova formulação para a função custo que permita incluir e tratar matematicamente a característica esparsa do vetor W. Tal formulação é encontrada na análise do algoritmo LMS com restrições (Frost, 1972). Assim, dentro desse 


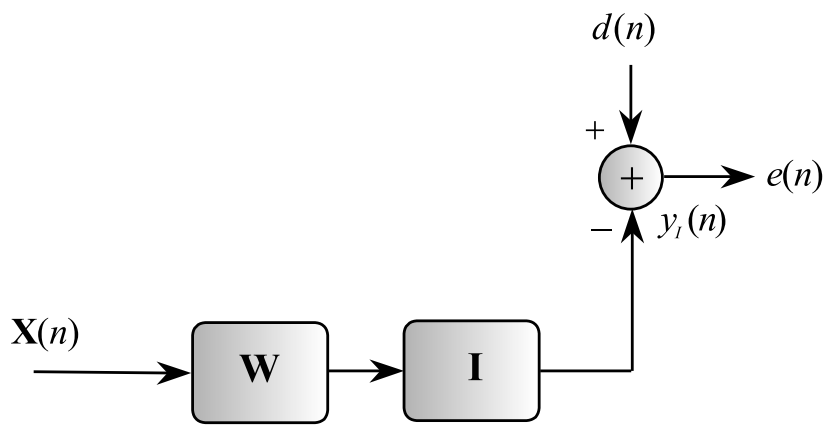

Figura 2: Diagrama em blocos para a determinação do vetor ótimo de coeficientes.

contexto, a função custo é dada por:

$$
\begin{gathered}
\operatorname{minimize} E\left[e^{2}(n)\right] \\
\text { sujeito a } \mathbf{C}^{T} \mathbf{W}=\mathbf{f}
\end{gathered}
$$

O fator de interpolação $(L)$ determina que $(L-1)$ coeficientes são fixados em zero entre dois coeficientes não nulos. Dessa forma, as dimensões da matriz de restrições $\mathbf{C}$ e do vetor de resposta às restrições $\mathbf{f}$ são $N \times L_{z}$ e $L_{z} \times 1$, respectivamente, onde $N \times 1$ é a dimensão de W e $L_{z}=\lfloor N / L\rfloor$ é o número de coeficientes com valor zero no vetor de coeficientes esparso. O operador $\lfloor\cdot\rfloor$ denota a operação de truncamento.

Com a utilização dos multiplicadores de Lagrange em (1) e (2), passamos de um problema de otimização com restrições para um problema clássico de otimização (Frost, 1972). Assim, a função custo é expressa por:

$$
J=E\left[e^{2}(n)\right]+\lambda^{T}\left(\mathbf{C}^{T} \mathbf{W}-\mathbf{f}\right) .
$$

Da Figura 2, o erro instantâneo é dado por:

$$
e(n)=d(n)-y(n)=d(n)-\mathbf{X}_{I}^{T}(n) \mathbf{W},
$$

onde

$$
\mathbf{X}_{I}(n)=\sum_{j=0}^{M-1} i_{j} \mathbf{X}(n-j)
$$

com $i_{j}$, para $j=0, \ldots, M-1$, representando os coeficientes do filtro interpolador. Elevando ao quadrado a equação (4) e tomando seu valor esperado, obtém-se:

$$
\begin{aligned}
E\left[e^{2}(n)\right]= & \sigma_{d}^{2}-2 \sum_{k=0}^{M-1} i_{k} E\left[d(n) \mathbf{X}^{T}(n-k)\right] \mathbf{W} \\
& +\sum_{k=0}^{M-1} \sum_{l=0}^{M-1} i_{k} i_{l} \mathbf{W}^{T} E[\mathbf{X}(n-k) \\
& \left.\times \mathbf{X}^{T}(n-l)\right] \mathbf{W} .
\end{aligned}
$$

Definindo $\mathbf{P}_{I}=\sum_{k=0}^{M-1} i_{k} E\left[d(n) \mathbf{X}^{T}(n-k)\right]$ e $\mathbf{R}_{I I}=$ $\sum_{k=0}^{M-1} \sum_{l=0}^{M-1} i_{k} i_{l} E\left[\mathbf{X}(n-k) \mathbf{X}^{T}(n-l)\right]$, a equação (3) é reescrita da seguinte forma:

$$
J=\sigma_{d}^{2}-2 \mathbf{P}_{I} \mathbf{W}+\mathbf{W}^{T} \mathbf{R}_{I I} \mathbf{W}+\lambda^{T}\left(\mathbf{C}^{T} \mathbf{W}-\mathbf{f}\right)
$$

O vetor de coeficientes ótimo é obtido a partir de $\frac{\partial J}{\partial \mathbf{W}}=0$. Seguindo o mesmo procedimento apresentado em Frost (1972), determina-se também o vetor de multiplicadores de Lagrange $\lambda$. Assim, o vetor de coeficientes ótimo, incorporando as restrições, é dado por:

$\mathbf{W}_{o t}=\mathbf{R}_{I I}^{-1} \mathbf{P}_{I}+\mathbf{R}_{I I}^{-1} \mathbf{C}\left(\mathbf{C}^{T} \mathbf{R}_{I I}^{-1} \mathbf{C}\right)^{-1}\left(\mathbf{f}-\mathbf{C}^{T} \mathbf{R}_{I I}^{-1} \mathbf{P}_{I}\right)$.

Note que a equação (8) é composta por dois termos. Um é o vetor ótimo sem restrições, obtido da forma clássica para uma estrutura AFIR, denotado por $\left.\mathbf{W}_{\text {ot }}\right|_{\text {nrestr }}=$ $\mathbf{R}_{I I}^{-1} \mathbf{P}_{I}$. O outro termo leva em consideração as restrições e modifica a solução irrestrita de forma a satisfazer a equação (2) (Resende et alii, 1996). Dessa forma, a equação (8) pode ser reescrita como:

$$
\begin{aligned}
\mathbf{W}_{\text {ot }}= & \left.\mathbf{W}_{\text {ot }}\right|_{\text {nrestr }}+\mathbf{R}_{I I}^{-1} \mathbf{C}\left(\mathbf{C}^{T} \mathbf{R}_{I I}^{-1} \mathbf{C}\right)^{-1} \\
& \times\left(\mathbf{f}-\left.\mathbf{C}^{T} \mathbf{W}_{\text {ot }}\right|_{\text {nrestr }}\right) .
\end{aligned}
$$

Neste ponto, é importante ressaltar que os valores esperados da matriz de autocorrelação de entrada, $\mathbf{R}_{I I}$, e o vetor de correlação cruzada entre o sinal de entrada e o sinal desejado, $\mathbf{P}_{I}$, são determinados sem aplicar a TI.

A Figura 3 mostra, para o caso de duas dimensões, as curvas de nível correspondentes à superfície de desempenho para o filtro $\mathbf{W}=\left[\begin{array}{ll}w_{1} & w_{2}\end{array}\right]^{T}$ completo (estrutura mostrada na Figura 2, considerando todos os coeficientes de $\mathbf{W})$. Nesse caso, os coeficientes ótimos são obtidos 
da expressão $\left.\mathbf{W}_{\text {ot }}\right|_{\text {nrestr }}=\mathbf{R}_{I I}^{-1} \mathbf{P}_{I}$, mostrado na Figura 3 por " $\times$ ". Observe que o vetor ótimo $\left.\mathbf{W}_{\text {ot }}\right|_{\text {nrestr }}$ corresponde à solução para o algoritmo LMS filtrado (Tobias et alii, 2000). Para o caso de $\mathbf{W}$ esparso $\left(\mathbf{W}=\left[\begin{array}{ll}w_{1} & 0\end{array}\right]^{T}\right)$, os coeficientes ótimos são dados pela equação (9) (representado por "o" na Figura 3). No caso esparso, a superfície de desempenho corresponde à interseção entre a parabolóide (superfície de desempenho considerando $\mathbf{W}$ não esparso) e o plano perpendicular ao plano $\left(w_{1}, w_{2}\right)$, para $w_{2}=0$, representado pela linha vertical central da Figura 3. Em outras palavras, a superfície de desempenho no caso esparso para duas dimensões é reduzida a uma parábola. Como se pode observar da Figura 3, as restrições impostas ao vetor $\mathbf{W}$ levam o erro mínimo nesse caso a ser maior do que aquele do caso de $\mathbf{W}$ não esparso.

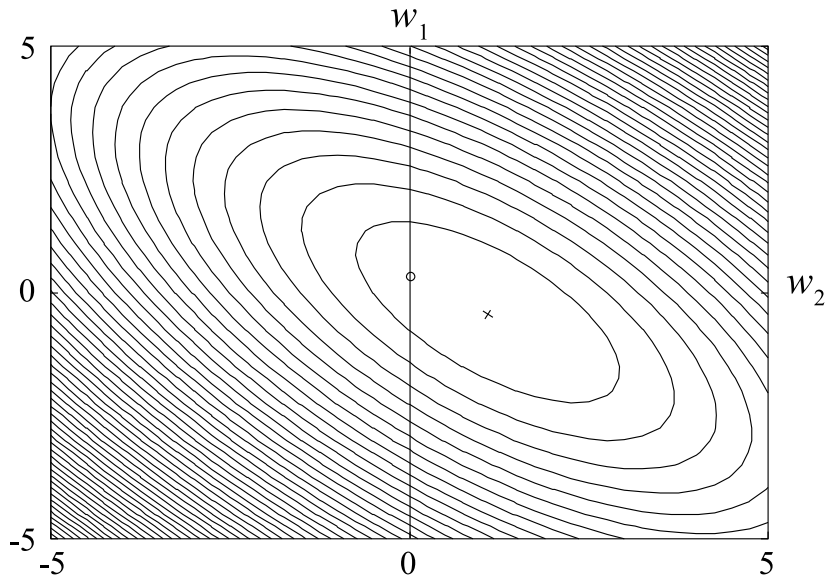

Figura 3: Coeficientes ótimos para o caso $\mathbf{W}$ esparso e não esparso.

\section{ALGORITMO LMS FILTRADO COM RESTRIÇÕES}

O objetivo desta seção é obter uma expressão analítica para a atualização do vetor de coeficientes esparso do filtro adaptativo, utilizando o algoritmo LMS. Para isso, vamos utilizar a aproximação estocástica da equação (3), ou seja, o cálculo do valor esperado é ignorado. Isso implica em usar a versão instantânea da função custo representada pela equação (3), levando à seguinte expressão:

$$
\hat{J}(n)=e^{2}(n)+\lambda^{T}(n)\left(\mathbf{C}^{T} \mathbf{W}(n)-\mathbf{f}\right) .
$$

A equação de atualização dos coeficientes do filtro adaptativo, que utiliza o gradiente estocástico, é dada por:

$$
\begin{aligned}
\mathbf{W}(n+1)= & \mathbf{W}(n)-\mu \frac{\partial \hat{J}(n)}{\partial \mathbf{W}(n)} \\
= & \mathbf{W}(n)+2 \mu e(n) \\
& \times \frac{\partial e(n)}{\partial \mathbf{W}(n)}-\mu \mathbf{C} \lambda(n)
\end{aligned}
$$

Com base na Figura 2, acrescentando-se um ruído de medição $z(n)$ ao sinal $d(n)$ e substituindo-se $\mathbf{W}$ por $\mathbf{W}(n)$, o sinal de erro é

$$
\begin{aligned}
e(n)= & d(n)-y(n)+z(n) \\
= & d(n)-\sum_{j=0}^{M-1} i_{j} \mathbf{X}^{T}(n-j) \\
& \times \mathbf{W}(n-j)+z(n) .
\end{aligned}
$$

Assim, tomando-se a derivada do erro instantâneo em relação ao vetor $\mathbf{W}(n)$, obtém-se a equação de atualização dos coeficientes, dada por:

$$
\mathbf{W}(n+1)=\mathbf{W}(n)+2 \mu e(n) \mathbf{X}_{I}(n)-\mu \mathbf{C} \lambda(n) .
$$

Na Figura 4(a), é mostrado o diagrama em blocos representando a equação (13). Uma característica importante, que deve ser observada, é o fato de que o algoritmo de adaptação (o LMS neste caso) tem, como sinal de entrada, a versão filtrada pelo interpolador do vetor $\mathbf{X}(n)$ de entrada, denotada por $\mathbf{X}_{I}(n)$. Isso implica que uma variante do algoritmo LMS deva ser usada, no caso o algoritmo LMS filtrado (Tobias et alii, 2000). A introdução das restrições decorre da característica esparsa de $\mathbf{W}(n)$. A estrutura da Figura 4(a) pode ser modificada a fim de se obter uma redução adicional no número de operações aritméticas da estrutura adaptativa (Seara et alii, 1992). Assumindo a condição de adaptação lenta, pode-se inverter a posição dos blocos correspondentes ao interpolador e o filtro esparso da Figura 4(a), resultando na Figura 4(b). Note que a estrutura modificada agora requer apenas um bloco interpolador. A notação utilizada (Figura 4(b)) é a seguinte: $\mathbf{W}(n)$ representa o vetor esparso adaptativo; $\mathbf{X}(n)$ e $\mathbf{X}_{I}(n)$ são os vetores de entrada e sua versão filtrada pelo interpolador, respectivamente; I é o vetor que caracteriza a resposta ao impulso do interpolator, sendo sua dimensão $M \times 1 ; \quad d(n), \quad y(n)$ e $e(n)$ representam, respectivamente, a saída desejada, a saída do filtro esparso e o 


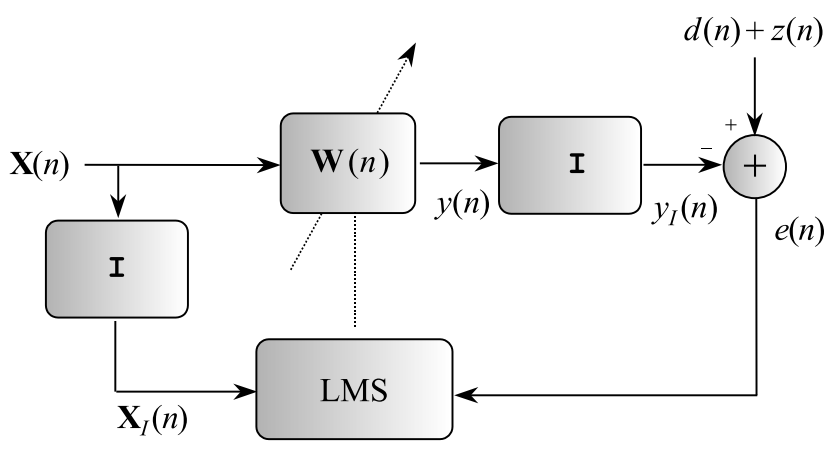

(a)

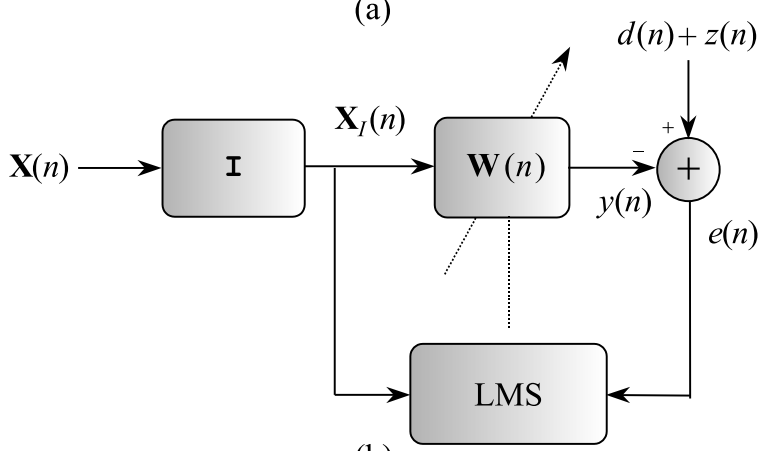

(b)

Figura 4: Diagrama em blocos das estruturas adaptativas: (a) estrutura direta; (b) estrutura modificada.

sinal de erro; $z(n)$ é um ruído branco, não correlacionado com nenhum outro sinal no sistema, de variância $\sigma_{z}^{2}$

Daqui em diante, será considerado apenas o esquema mostrado na Figura 4(b) (estrutura modificada). O sinal de erro obtido a partir dessa estrutura é dado por:

$e(n)=d(n)-y(n)+z(n)=d(n)-\mathbf{X}_{I}^{T}(n) \mathbf{W}(n)+z(n)$.

Note que a equação (14) pode ser obtida a partir da equação (12) através da consideração de que $\mathbf{W}(n-$ $j) \approx \mathbf{W}(n)$ para $j=0, \cdots, M-1$. O que é consistente com a suposição de adaptação lenta. Seguindo o mesmo procedimento anterior e determinando $\lambda(n)$ como em Frost (1972), a equação de atualização dos coeficientes do filtro adaptativo é dada por:

$$
\begin{aligned}
\mathbf{W}(n+1)= & \mathbf{F}\left(\mathbf{W}(n)-2 \mu \mathbf{X}_{I}(n) \mathbf{X}_{I}^{T}(n) \mathbf{W}(n)\right. \\
& \left.+2 \mu d(n) \mathbf{X}_{I}(n)+2 \mu z(n) \mathbf{X}_{I}(n)\right)+\mathbf{q}
\end{aligned}
$$

onde $\mathbf{F}=\mathbf{I}-\mathbf{C}\left(\mathbf{C}^{T} \mathbf{C}\right)^{-1} \mathbf{C}^{T}$ e $\mathbf{q}=\mathbf{C}\left(\mathbf{C}^{T} \mathbf{C}\right)^{-1} \mathbf{f}$.

\section{VALOR ESPERADO DO VETOR DE COEFICIENTES}

Nesta seção, vamos determinar uma expressão teórica que permita predizer o comportamento médio dos coeficientes do filtro esparso adaptativo. Tomando o valor esperado de (15), obtém-se a seguinte expressão:

$$
\begin{aligned}
E[\mathbf{W}(n+1)]= & \mathbf{F}\left(E[\mathbf{W}(n)]-2 \mu E\left[\mathbf{X}_{I}(n)\right.\right. \\
& \left.\times \mathbf{X}_{I}^{T}(n) \mathbf{W}(n)\right]+2 \mu E[d(n) \\
& \left.\left.\times \mathbf{X}_{I}(n)\right]+2 \mu E\left[z(n) \mathbf{X}_{I}(n)\right]\right)+\mathbf{q}
\end{aligned}
$$

Com base em exaustivos resultados de simulações, apresentados em Tobias (1999), consideremos a seguinte hipótese simplificativa:

$$
E\left[\mathbf{X}_{I}(n) \mathbf{X}_{I}^{T}(n) \mathbf{W}(n)\right] \approx E\left[\mathbf{X}_{I}(n) \mathbf{X}_{I}^{T}(n)\right] E[\mathbf{W}(n)]
$$

onde,

$$
\begin{aligned}
E\left[\mathbf{X}_{I}(n) \mathbf{X}_{I}^{T}(n)\right] & =\sum_{k=0}^{M-1} \sum_{l=0}^{M-1} i_{k} i_{l} E\left[\mathbf{X}(n-k) \mathbf{X}^{T}(n-l)\right] \\
& =\sum_{k=0}^{M-1} \sum_{l=0}^{M-1} i_{k} i_{l} \mathbf{R}_{k-l}=\mathbf{R}_{I I}
\end{aligned}
$$

Através da suposição estabelecida pela equação (17), estamos assumindo que a correlação entre os vetores de entrada é mais importante do que a correlação entre os vetores de entrada e o vetor de coeficientes do filtro adaptativo. É importante observar que a aplicação da teoria da independência (Mazo, 1979) em (18) determina que $E\left[\mathbf{X}(n-k) \mathbf{X}^{T}(n-l)\right]=0$ para $k \neq l$. Portanto, as equações (17) e (18) estão claramente em oposição a tal suposição. Na seção correspondente aos resultados experimentais, são mostradas as curvas obtidas a partir dos modelos, considerando e não considerando a teoria da independência. Pode ser observado que este último modelo descreve bem melhor o comportamento médio dos coeficientes do filtro adaptativo. Assim, substituindo-se (17) em (16) e manipulando-se adequadamente os termos, obtém-se a seguinte expressão:

$$
E[\mathbf{W}(n+1)]=\left(\mathbf{F}-2 \mu \mathbf{F} \mathbf{R}_{I I}\right) E[\mathbf{W}(n)]+2 \mu \mathbf{F} \mathbf{P}_{I}+\mathbf{q},
$$

onde o valor esperado do termo contendo $z(n)$ é zero pelas considerações anteriormente estabelecidas. 


\subsection{Vetor de erro nos coeficientes}

Definindo-se o vetor de erro nos coeficientes, como sendo $\mathbf{V}(n)=\mathbf{W}(n)-\mathbf{W}_{\text {ot }}$, e utilizando-se a equação (15), a expressão recursiva, para obter esse vetor, pode ser escrita da seguinte forma:

$$
\begin{aligned}
\mathbf{V}(n+1)= & \mathbf{F}\left(\mathbf{I}-2 \mu \mathbf{X}_{I}(n) \mathbf{X}_{I}^{T}(n)\right) \mathbf{V}(n) \\
& +2 \mu \mathbf{F} d(n) \mathbf{X}_{I}(n)-2 \mu \mathbf{X}_{I}(n) \mathbf{X}_{I}^{T}(n) \mathbf{W}_{o t} \\
& +2 \mu \mathbf{F} z(n) \mathbf{X}_{I}(n)+(\mathbf{F}-\mathbf{I}) \mathbf{W}_{\text {ot }}+\mathbf{q} . \quad(20)
\end{aligned}
$$

Aplicando-se (17) e utilizando-se a relação dada pela equação (8), o valor esperado da equação (20) é

$$
E[\mathbf{V}(n+1)]=\mathbf{F}\left(\mathbf{I}-2 \mu \mathbf{R}_{I I}\right) E[\mathbf{V}(n)]
$$

Considerando-se que a matriz $\mathbf{F}$ é tal que $\mathbf{F}^{2}=\mathbf{F}$ (propriedade da idempotence) (Frost, 1972), podemos então reescrever a equação (21) como segue:

$$
E[\mathbf{V}(n+1)]=\left(\mathbf{I}-2 \mu \mathbf{F R}_{I I} \mathbf{F}\right) E[\mathbf{V}(n)]
$$

Através do exemplo a seguir, pode-se observar a forma como é construída a matriz de restrições. Assim, considerando-se $L=2$ e um filtro adaptativo com 5 coeficientes, a matriz de restrições $\mathbf{C}$ e o vetor resposta f assumem, respectivamente, as seguintes formas:

$$
\mathbf{C}=\left(\begin{array}{ll}
0 & 0 \\
1 & 0 \\
0 & 0 \\
0 & 1 \\
0 & 0
\end{array}\right) \text { e } \mathbf{f}=\left(\begin{array}{l}
0 \\
0
\end{array}\right)
$$

$\mathrm{Na}$ matriz $\mathbf{C}$, os elementos com valor "1" determinam a posição dos coeficientes do filtro adaptativo que não são adaptados e o vetor $\mathbf{f}$ indica os valores para os quais eles são fixados.

\section{RESULTADOS EXPERIMENTAIS}

Nesta seção, a qualidade do modelo proposto é avaliada através de simulações numéricas. Para isso, são utilizados dois tipos de processos de entrada, ruído branco e colorido, aplicados a um caso de identificação de sistemas. O valor utilizado para a taxa de convergência está relacionado ao máximo valor permitido, para o qual o algoritmo apresenta convergência. Para todos os exemplos, a resposta ao impulso do filtro interpolador é dada por $[0,5 ; 1 ; 0,5]$, o fator de interpolação é $L=2$, e os sinais de entrada e ruído aditivo têm variâncias 1 e 0,0001, respectivamente.

Exemplo 1: A resposta ao impulso da planta é dada por $[1 ; 0,8 ; 0,6 ; 0,1 ;-0.2]$, o sinal de entrada é um ruído branco, e o valor máximo para a taxa de convergência é $\mu_{\max }=0,08$ (determinado de forma experimental). Os valores utilizados para a taxa de convergência são: $0,2 \mu_{\max }$ e $0,06 \mu_{\max }$. A Figura 5 mostra os resultados obtidos por simulação (média de 50 realizações): modelo clássico, utilizando a TI e modelo proposto (equação (19)). Nessa figura, pode-se verificar a predição do valor em regime permanente para os coeficientes do filtro esparso, dado pela equação (9), sendo $\mathbf{W}_{\text {ot }}=[1,06 ; 0 ; 0,03 ; 0 ;-0,07]$. Pode-se observar que, para valores de $\mu$ próximos do máximo valor permitido, o modelo proposto apresenta um pequeno descasamento com relação aos resultados obtidos por simulação (Figura 5(a)). Esse comportamento é esperado, uma vez que o modelo foi obtido a partir da suposição de adaptação lenta. Na medida em que se reduz o valor de $\mu$, os resultados de simulação e os resultados via modelo proposto apresentam um casamento muito bom, como mostrado na Figura 5(b). Por outro lado, pode-se observar que o modelo que utiliza a TI não representa adequadamente o comportamento médio dos coeficientes para qualquer que seja o valor de $\mu$.

Exemplo 2: Para esse exemplo, foi utilizada a mesma planta do Exemplo 1. Nesse caso, o sinal de entrada é um ruído colorido gerado a partir de um processo AR de segunda ordem, dado por $x(n)=a_{1} x(n-1)+$ $a_{2} x(n-2)+u(n)$, onde $u(n)$ é um ruído branco com variância 0,0322 . Os coeficientes do processo AR são: $a_{1}=-1,5955$ e $a_{2}=0,95$, e a dispersão dos autovalores é de 10 . O valor máximo para a taxa de convergência é $\mu_{\max }=0,09$. Similarmente ao exemplo anterior, são utilizados os valores $0,2 \mu_{\max }$ e 0,06 $\mu_{\max }$. A Figura 6 mostra as curvas obtidas por simulação (média de 50 realizações), utilizando o modelo com a TI e o modelo proposto pela equação (19). A partir da equação (9), obtém-se o valor em regime permanente para os coeficientes do filtro esparso, que são: $\mathbf{W}_{o t}=[1,27 ; 0 ;-0,19 ; 0 ; 0,007]$, e podem ser verificados nesta mesma figura. Novamente, observa-se que o modelo proposto apresenta melhor casamento para valores de taxa de convergência que satisfazem à condição de adaptação lenta. Da mesma forma que no caso de entrada ruído branco, para um sinal de entrada colorido, a predição via modelo teórico e sua respectiva simulação também apresentam uma excelente concordância.

Exemplo 3: Nesse exemplo, foi utilizada uma planta

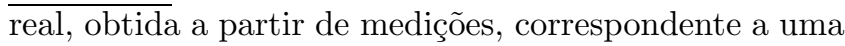
híbrida de um canal telefônico para transmissão de da- 


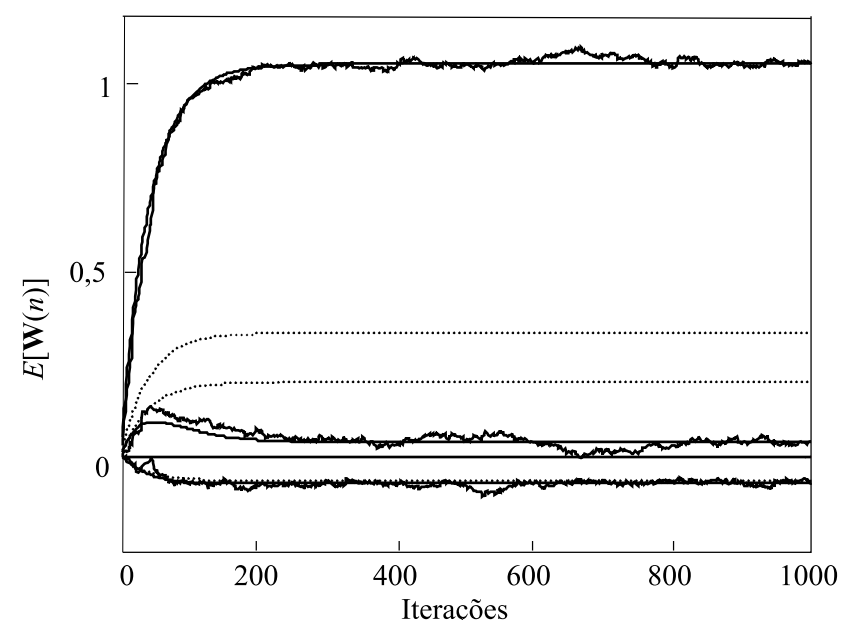

(a)

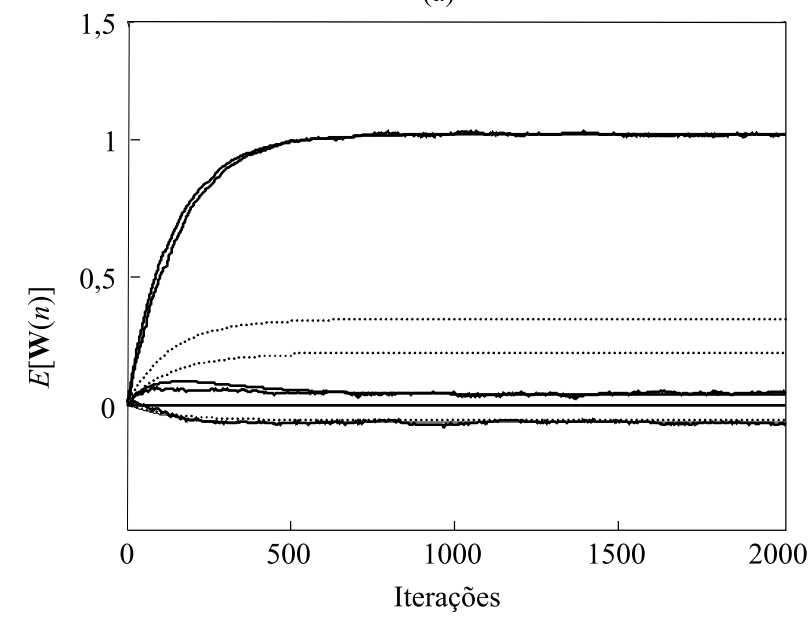

(b)

Figura 5: Evolução de $E[\mathbf{W}(n)]$ para o Exemplo 1. (a) $\mu=0,2 \mu_{\max }$; (b) $\mu=0,06 \mu_{\max }$. Linha irregular representa a simulação (média de 50 realizações); linha pontilhada, o modelo usando a TI; linha cheia, o modelo proposto (equação (19)).

dos. Sua resposta ao impulso é mostrada na Figura $7(\mathrm{a})$. O sinal de entrada do sistema adaptativo é um ruído branco com variância unitária. O valor máximo para a taxa de convergência é $\mu_{\max }=0,01$. Neste exemplo, utilizamos 0,2 $\mu_{\max }$. A Figura 7(b) mostra as curvas obtidas por simulação (média de 50 realizações) e pelo modelo proposto (equação (19)). A fim de manter o gráfico o mais claro possível, as curvas do modelo que utiliza a TI são apresentadas em figura separada (Figura $7(\mathrm{c}))$. A partir das Figuras $7(\mathrm{~b})$ e $7(\mathrm{c})$, pode-se verificar o bom desempenho do modelo proposto para um número elevado de coeficientes. Ao mesmo tempo, é possível observar que o modelo que utiliza a TI apresenta uma pobre predição da evolução dos coeficientes do filtro adaptativo.

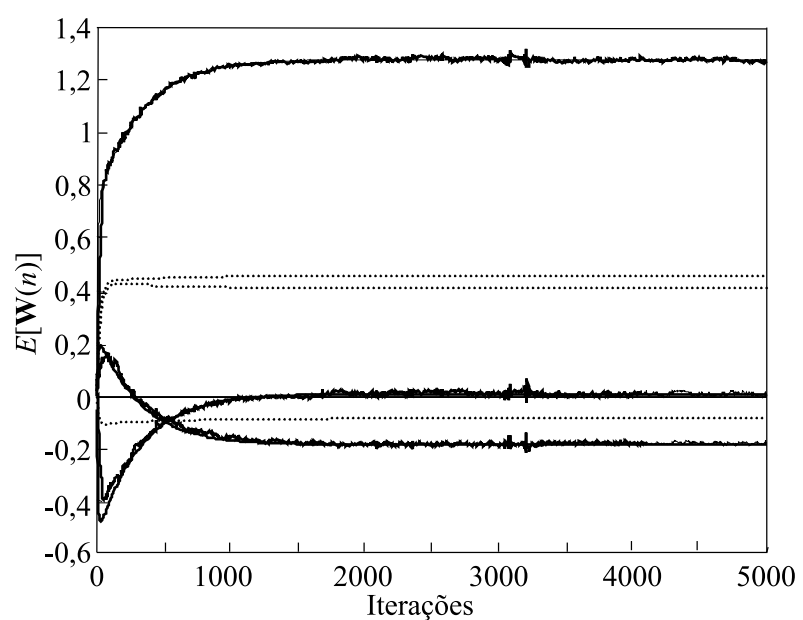

(a)

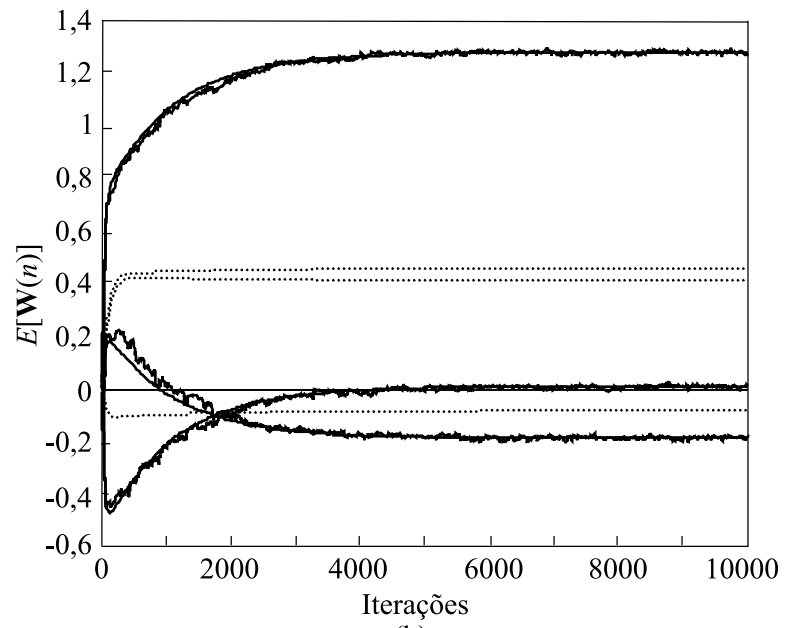

(b)

Figura 6: Evolução de $E[\mathbf{W}(n)]$ para o Exemplo 2. (a) $\mu=0,2 \mu_{\max }$; (b) $\mu=0,06 \mu_{\max }$. Linha irregular representa a simulação (média de 50 realizações); linha pontilhada, o modelo usando a TI; linha cheia, o modelo proposto (equação (19)).

\section{CONCLUSÕES}

A estrutura particular dos filtros AIFIR-LMS faz com que o procedimento de análise clássica, amplamente utilizado para a análise de estruturas adaptativas FIRLMS, já não possa mais ser aplicável. Dessa forma, uma versão filtrada do algoritmo CLMS (Frost, 1972) é desenvolvida. Através da aplicação de uma análise com restrições, é possível tratar matematicamente a estrutura de um filtro adaptativo IFIR e, juntamente com a não utilização da teoria da independência, obter uma excelente predição do valor em regime permanente dos coeficientes do filtro esparso, como também um modelo analítico para descrever seu comportamento em regime transitório. 


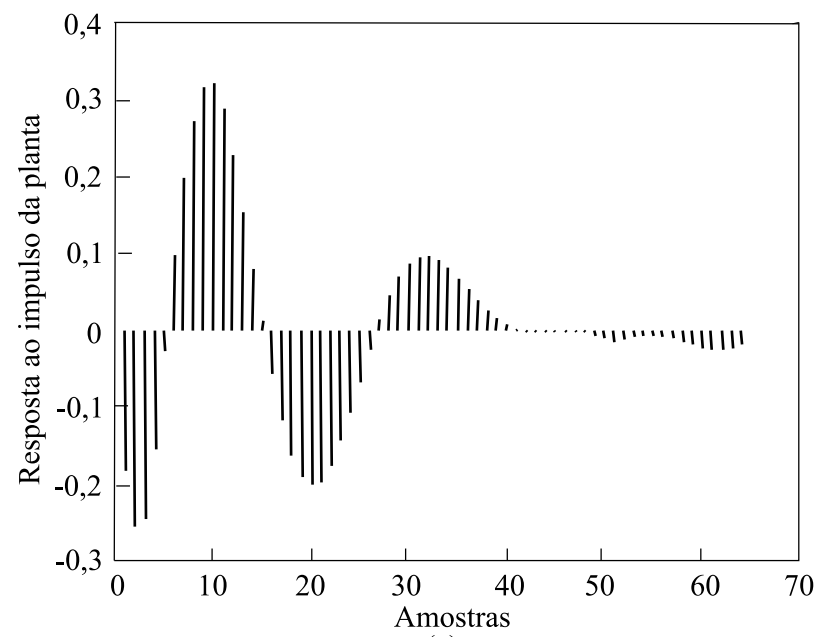

(a)

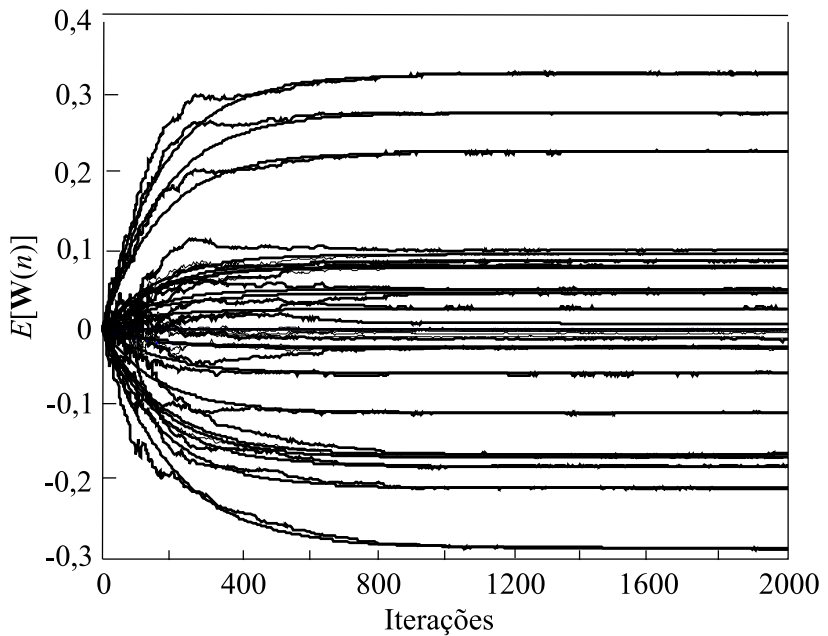

(b)

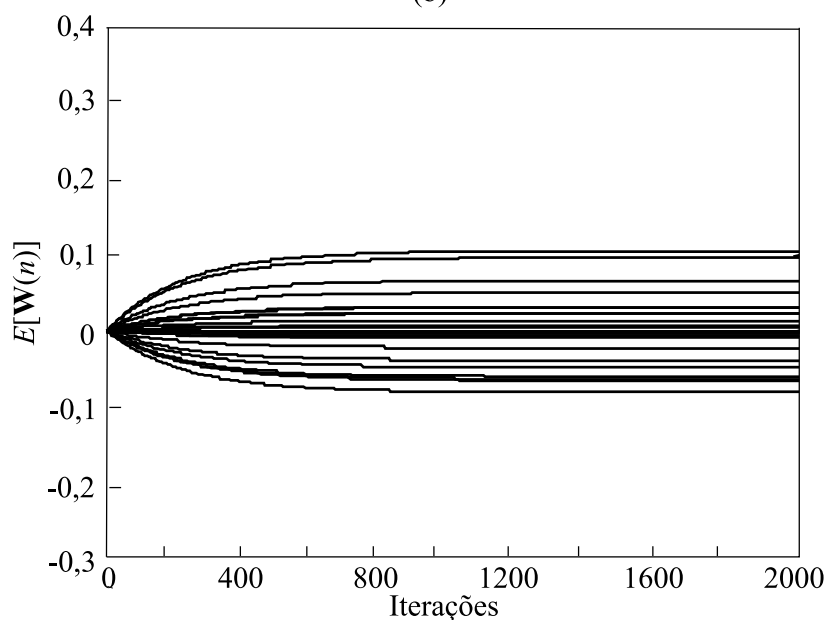

(c)

Figura 7: Exemplo 3. (a) Resposta ao impulso de uma híbrida de um canal telefônico para transmissão de dados; (b) evolução de $E[\mathbf{W}(n)]$ : linha irregular representa a simulação (média de 50 realizações); linha cheia, o modelo proposto, (equação (19)); (c) modelo para $E[\mathbf{W}(n)]$, usando a TI.

\section{REFERÊNCIAS}

Abousaada, A., T. Aboulnasr and W. Steenaart (1992). "An echo tail canceller based on adaptive interpolated FIR Filtering". IEEE Trans. Circuits Syst.-II, Vol. 39, pp. 409-416.

Frost III, O. L. (1972). "An algorithm for linearly constrained adaptive array processing". Proc. IEEE, Vol. 60, n. 8, pp. 926-935.

Haykin, S. (1986). Adaptive Filter Theory. PrenticeHall, Englewood Cliffs, NJ.

Mazo, J. E. (1979). "On the independence theory of equalizer convergence". Bell Syst. Tech. J., Vol. 58, pp. 963-993.

Neuvo, Y., C. Y. Dong and S. K. Mitra (1984). "Interpolated finite impulse response digital filters". IEEE Trans. Acoust., Speech, Signal Processing, Vol. 32, pp. $563-570$.

Neuvo, Y., G. Rajan and S. K. Mitra (1987). "Design of narrow-band FIR bandpass digital filters with reduced arithmetic complexity". IEEE Trans. Circuits Syst., Vol. 34, pp. 409-419.

Resende, L. S., J. T. Romano and M. G. Bellanger (1996). "A fast least-squares algorithm for linearly constrained adaptive filtering". IEEE Trans. on Signal Proc., Vol. 44, pp. 1168-1174.

Seara, R., J. C. M. Bermudez and E. Beck (1992). "A new technique for the implementation of adaptive IFIR filters". Proc. ISSSE'92, Paris, France, pp. 644-647.

Saramäki, T., Y. Neuvo and S. K. Mitra (1988). "Design of computationally efficient interpolated FIR filters". IEEE Trans. Circuits Syst., Vol. 35, pp. $70-88$.

Tobias, O. J. (1999). "Análise estatística do algoritmo LMS filtrado". Tese (Doutorado em Engenharia Elétrica) - Centro Tecnológico, Universidade Federal de Santa Catarina, Florianópolis - SC.

Tobias, O. J., J. C. M. Bermudez and N. Bershad (2000). "Mean weight behavior of the filteredX LMS algorithm". IEEE Trans. on Signal Proc., Vol. 48, pp. 1061-1075.

Widrow, B. and S. Stearns (1985). Adaptive Signal Processing. Prentice-Hall. 